Vietnam Journal of Mechanics, NCST of Vietnam Vol. 24, 2002, No 1 (25 - 34)

\title{
A REDUCED FORM OF SHAKEDOWN KINEMATIC THEOREM
}

\author{
Pham Duc Chinh \\ Institute of Mechanics, NCST of Vietnam
}

\begin{abstract}
A reduced form of the shakedown kinematic theorem without time integrals is deduced for Tresca material, which is equivalent to the original one when the principal directions of plastic deformations everywhere in astructure remain unchanged during loading cycles.
\end{abstract}

\section{Introduction}

An elastio-perfectly plastic body subjected to loading cycles, though not undergoing instantaneous plastic collapse, may fail because plastic deformations accumulated during cycles increase indefinitely (leading to incremental collapse) or change signs endlessly (leading to low cycle fatigue). On the other hand, it may happen that no further plastic deformation occurs after one or a few cycles - that is, all subsequent cycles are elastic. In that case the body is said to shake down. The shakedown (quasistatic) theory, which contains the plastic limit theory ([1], [2]) as its limit case, has been comprehensively presented in the classical work of Koiter [3] and is naturally extended for general dynamic processes ([4], [5], [6]). The primary kinematic theorem formulated by Koiter is difficult to be used because of its complexity caused by the presence of time integrals. To eliminate them, Gokhfeld [7] and Sawczuk [8] (see also [9]) have restricted admissible plastic deformation cycles to a special proportional monotonous deformation mode (called the perfect incremental collapse one), and obtained subsequently an upper bound on the shakedown safety factor. With a broader admissible set of proportional (but need not to be monotonous) plastic deformations at every point $\mathrm{x} \in V$, we [10] succeeded in deducing a better bound on the shakedown factor. Other reduced forms (without time integrals) of the kinematic theorem for certain problems are obtained in [11], [12]. Let $\sigma^{e}(\mathbf{x}, t)$ denote the fictitious stress response of the body $V(\mathbf{x} \in V)$ to external agencies over a period of time $t \in[0, T]$ in assumption of its perfectly elastic behaviour. We call it a loading process. A set of loading processes is given as $\mathcal{L}_{t}: \sigma^{e} \in \mathcal{L}_{t}$. The values of all $\sigma^{e} \in \mathcal{L}_{t}$ are supposed to belong to a bounded time-independent loading domain $\mathcal{L}$ in the stress space over $V, \mathbf{e}^{p}(\mathbf{x}, t)$ is plastic strain rate. Following Koiter, define a set $\mathcal{A}$ of admissible plastic strain rate cycles: 


$$
\mathcal{A}=\left\{\mathrm{e}^{p} \in \mathcal{E} \mid \varepsilon^{p}=\int_{0}^{T} \mathrm{e}^{p} d t \in \mathcal{C}\right\} .
$$

Let $D\left(\mathbf{e}^{p}\right)$ denote the dissipation function. For Tresca material it has the particular form

$$
D\left(\mathbf{e}^{p}\right)=\boldsymbol{\sigma}: \mathbf{e}^{p}=\frac{1}{2} \sigma_{Y}\left(\left|e_{1}^{p}\right|+\left|e_{2}^{p}\right|+\left|e_{3}^{p}\right|\right),
$$

where $\sigma_{Y}$ is the yield stress in tension, $e_{1}^{p}, e_{2}^{p}$ and $e_{3}^{p}$ are the principal components of the plastic strain rate tensor $\mathbf{e}^{p}$.

Koiter's shakedown kinematic theorem can be stated as $[9,10]$

$$
k_{s}^{-1}=\sup _{\mathbf{e}^{p} \in \mathcal{A} ; \sigma^{e} \in \mathcal{L}_{t}} \frac{\int_{0}^{T} d t \int_{V} \sigma^{e}: \mathbf{e}^{p} d V}{\int_{0}^{T} d t \int_{V} D\left(\mathrm{e}^{p}\right) d V}
$$

(at $k_{s}>1$ the body will shake down, while it will not otherwise).

\section{A reduced theorem for Tresca material}

Our objective is to eliminate the time integrals in (1.3). If the plastic deformations at every point of a structure should be constrained to be proportional (i.e. confined to a certain direction in the strain space), then a reduced formulation without time integrals, which is equivalent to (1.3) can be deduced [10]. In this work we consider a larger class of problems for Tresca material: the direction of plastic deformations at every point in $V$ during cycles may change continuously in the strain space, however the principal directions of the plastic strains are fixed. In practice, the Tresca criterion is prefered over the Mises one in such cases where the principal directions of plastic deformations at every point in $V$ can be foreseen and fixed, then the use of Tresca criterion should be much simpler. Let these principal directions to be

$$
\varepsilon^{1}(\mathbf{x}), \varepsilon^{2}(\mathbf{x}), \varepsilon^{3}(\mathbf{x}), \quad \varepsilon^{i}(\mathbf{x}) \cdot \varepsilon^{j}(\mathbf{x})=\delta_{i j}, \quad i, j=1,2,3, \mathbf{x} \in V,
$$

$\delta_{i j}$ is usual Kronecker delta. Then the plastic strain rate $\mathbf{e}^{p}(\mathbf{x}, t)$ is presumed to have the form (components $e_{i}$ can be considered as principal deformations)

$$
\begin{aligned}
\mathbf{e}^{p}(\mathbf{x}, t) & =\sum_{i=1}^{3} e_{i}(\mathbf{x}, t) \varepsilon^{i}(\mathbf{x}), \quad \mathbf{x} \in V, \quad t \in[0, T] \\
\varepsilon^{p}(\mathbf{x}) & =\int_{0}^{T} \mathrm{e}^{p} d t=\sum_{i=1}^{3} \varepsilon_{i}(\mathbf{x}) \varepsilon^{i}(\mathbf{x}) .
\end{aligned}
$$


Our goal is to eliminate the time integrals in (1.3) for the class of problems restricted by (2.2). The dissipation function for Tresca material has the particular
form

$$
\begin{aligned}
& D\left(\mathbf{e}^{p}\right)=\frac{1}{2} \sigma_{Y}\left(\left|e_{1}\right|+\left|e_{2}\right|+\left|e_{3}\right|\right), \\
& D\left(\varepsilon^{p}\right)=\frac{1}{2} \sigma_{Y}\left(\left|\varepsilon_{1}\right|+\left|\varepsilon_{2}\right|+\left|\varepsilon_{3}\right|\right),
\end{aligned}
$$

$\sigma_{Y}$ - is the yield stress in tension-compression.

Because the Tresca material is plastically incompressible, one has

$$
e_{1}(\mathbf{x}, t)+e_{2}(\mathbf{x}, t)+e_{3}(\mathbf{x}, t)=0
$$

so at every point $\mathbf{x} \in V$, it is possible to divide the interval $[0, T]$ into three separated parts $T_{x}^{1}, T_{x}^{2}, T_{x}^{3}$ that (in each part - the respective two from the three scalars $e_{1}$, $e_{2}, e_{3}$ have the same sign)

$$
[0, T]=T_{x}^{1} \cup T_{x}^{2} \cup T_{x}^{3}, \quad T_{x}^{i} \cap T_{x}^{j}=\emptyset, i \neq j
$$

and

$$
e_{i}(\mathbf{x}, t) \cdot e_{j}(\mathbf{x}, t) \geq 0 \text { at } t \in T_{x}^{k}, \quad i \neq j \neq k \neq i .
$$

Note that $T_{x}^{k}$ may be not just one but a set of many intervals in $[0, T]$.

With (2.3)-(2.6) the dissipation function now has the particular form

$$
D\left(\mathrm{e}^{p}\right)=\sigma_{Y}\left(\left|e_{i}\right|+\left|e_{j}\right|\right), \quad t \in T_{x}^{k}, \quad i \neq j \neq k \neq i .
$$

Denote (components $\sigma_{i}$ are not principal stresses)

$$
\int_{T_{x}^{k}} e_{i}(\mathbf{x}, t) d t=\varepsilon_{i}^{k}(\mathbf{x}), \quad \sigma_{i}(\mathbf{x}, t)=\sigma^{e}(\mathbf{x}, t): \varepsilon^{i}(\mathbf{x}) .
$$

Then we have

$$
\begin{aligned}
& \varepsilon_{i}(\mathbf{x})=\int_{0}^{T} e_{i}(\mathbf{x}, t) d t=\varepsilon_{i}^{1}(\mathbf{x})+\varepsilon_{i}^{2}(\mathbf{x})+\varepsilon_{i}^{3}(\mathbf{x}), \\
& \boldsymbol{\sigma}^{e}: \mathbf{e}^{p}=\left(\sigma_{i}-\sigma_{k}\right) e_{i}+\left(\sigma_{j}-\sigma_{k}\right) e_{j}, \quad t \in T_{x}^{k}, \quad i \neq j \neq k \neq i .
\end{aligned}
$$

With (2.7)-(2.9), (1.3) can be rewritten as $(i \neq j \neq k \neq i)$

$$
k_{s}^{-1}=\sup _{e^{p} \in \mathcal{A} ; \sigma^{e} \in \mathcal{L}_{t}} \frac{\sum_{k=1}^{3} \int_{V} d \mathbf{x} \int_{T_{x}^{k}}\left[\left(\sigma_{i}-\sigma_{k}\right) e_{i}+\left(\sigma_{j}-\sigma_{k}\right) e_{j}\right] d t}{\sigma_{Y} \sum_{k=1}^{3} \int_{V} d \mathbf{x} \int_{T_{x}^{k}}\left(\left|e_{i}\right|+\left|e_{j}\right|\right) d t} .
$$


Any $\mathbf{e}^{p}$ from (2.2), (2.4)-(2.6), (2.8) can be decomposed into (the aim is to extract the time-dependent parts to integrate the time integrals in (2.10))

$$
e_{i}(\mathbf{x}, t)=\Lambda_{i}(\mathbf{x}, t)\left[\varepsilon_{i}^{k}(\mathbf{x})+\varepsilon_{i}^{k 0}(\mathbf{x})\right], \quad t \in T_{x}^{k}, \quad i \neq k,
$$

where

$$
\begin{gathered}
\varepsilon_{i}^{k 0}(\mathbf{x})=0 \quad \text { if } \quad \mathbf{x} \in V_{i}^{k}=\left\{\mathbf{x} \in V \mid \varepsilon_{i}^{k}(\mathbf{x}) \neq 0\right\} \\
\text { (in addition, denote } \left.\quad V_{i}^{k 0}=\left\{\mathbf{x} \in V \mid \varepsilon_{i}^{k}(\mathbf{x})=0\right\}\right) ; \\
\int_{T_{x}^{k}} \Lambda_{i} d t=1, \quad \int_{T_{x}^{k}}\left(\left|\Lambda_{i}\right|-\Lambda_{i}\right) d t=S_{i}^{k}(\mathbf{x}), \quad \mathbf{x} \in V_{i}^{k}, \\
\int_{T_{x}^{k}} \Lambda_{i} d t=0, \quad \int_{T_{x}^{k}}\left|\Lambda_{i}\right| d t=1, \quad \mathbf{x} \in V_{i}^{k 0} .
\end{gathered}
$$

Functions $\varepsilon_{i}^{k 0}(\mathbf{x})$ and $\Lambda_{i}(\mathbf{x}, t)$ are restricted by $(2.12),(2.13)$, but otherwise are arbitrary. $S_{i}^{k}(\mathbf{x}) / 2$ measures the absolute value of the integral of $\Lambda_{i}(\mathbf{x}, t)$ over the time intervals in $T_{i}^{k}$, during which $\Lambda_{i}(\mathbf{x}, t)$ is negative. Clearly $S_{i}^{k}(\mathbf{x})$ can take all possible positive values. One can verify

$$
\begin{aligned}
& \int_{T_{x}^{k}}\left(\left|\Lambda_{i}\right|+\Lambda_{i}\right) d t=S_{i}^{k}+2, \quad \int_{T_{x}^{k}}\left|\Lambda_{i}\right| d t=S_{i}^{k}+1, \quad \mathbf{x} \in V_{i}^{k} \\
& \int_{T_{x}^{k}}\left(\left|\Lambda_{i}\right|+\Lambda_{i}\right) d t=1, \quad \int_{T_{x}^{k}}\left(\left|\Lambda_{i}\right|-\Lambda_{i}\right) d t=1, \quad \mathbf{x} \in V_{i}^{k 0} .
\end{aligned}
$$

Denote $(i \neq k)$ :

$$
\begin{aligned}
\max _{t \in T_{x}^{k}}\left[\sigma_{i}(\mathbf{x}, t)-\sigma_{k}(\mathbf{x}, t)\right]\left[\varepsilon_{i}^{k}+\varepsilon_{i}^{k 0}\right] & =\left[\sigma_{i}\left(\mathbf{x}, t_{x k}^{i+}\right)-\sigma_{k}\left(\mathbf{x}, t_{x k}^{i+}\right)\right]\left[\varepsilon_{i}^{k}+\varepsilon_{i}^{k 0}\right] \\
& =\sigma_{i k}^{+}(\mathbf{x})\left[\varepsilon_{i}^{k}(\mathbf{x})+\varepsilon_{i}^{k 0}(\mathbf{x})\right], \\
\min _{t \in T_{x}^{k}}\left[\sigma_{i}(\mathbf{x}, t)-\sigma_{k}(\mathbf{x}, t)\right]\left[\varepsilon_{i}^{k}+\varepsilon_{i}^{k 0}\right] & =\left[\sigma_{i}\left(\mathbf{x}, t_{x k}^{i-}\right)-\sigma_{k}\left(\mathbf{x}, t_{x k}^{i-}\right)\right]\left[\varepsilon_{i}^{k}+\varepsilon_{i}^{k 0}\right] \\
& =\sigma_{i k}^{-}(\mathbf{x})\left[\varepsilon_{i}^{k}(\mathbf{x})+\dot{\varepsilon}_{i}^{k 0}(\mathbf{x})\right],
\end{aligned}
$$

here for short we have introduced new functions $\sigma_{i k}^{+}(\mathbf{x}), \sigma_{i k}^{-}(\mathbf{x})$, the meanings of which are clear from the context $\left(\sigma_{i k}^{+}, \sigma_{i k}^{-}\right.$are not components of the stress tensor); $t_{x k}^{i+}$ and $t_{x k}^{i-}$ denote the time instants, at which the corresponding maximums and minimums are reached: they are not unique because $\sigma^{e}(\mathbf{x}, t)$ of a process is arbitrary in $\mathcal{L}$ and 
may be repeated over times and we can choose $t_{x k}^{i+}, t_{x k}^{i-}$ to be distinct values for all $k$ and $i$.

Substituting (2.11) into (2.10) and taking into account (2.12)-(2.14) we get (the kinematical variables are understood to be restricted by $(1.1),(2.2)$ implicitly, while $\boldsymbol{\sigma}^{e} \in \mathcal{L}, \sum_{i \neq k}$ denotes the sum over $i=1,2,3$ that $i \neq k$ )

$$
k_{s}^{-1}=\sup _{\varepsilon_{i}^{k}, \varepsilon_{i}^{k 0}, \Lambda_{i}} \frac{\sum_{k=1}^{3} \sum_{i \neq k} \int_{V} d \mathbf{x} \int_{T_{x}^{k}}\left(\sigma_{i}-\sigma_{k}\right)\left(\varepsilon_{i}^{k}+\varepsilon_{i}^{k 0}\right)\left(\frac{\left|\Lambda_{i}\right|+\Lambda_{i}}{2}-\frac{\left|\Lambda_{i}\right|-\Lambda_{i}}{2}\right) d t}{\sigma_{Y} \sum_{k=1}^{3} \sum_{i \neq k} \int_{V} d x \int_{T_{x}^{k}}\left|\Lambda_{i}\right|\left|\varepsilon_{i}^{k}+\varepsilon_{i}^{k 0}\right| d t} .
$$

Now from (2.12)-(2.14) one can verify

$$
k_{s}^{-1} \leq \sup _{\varepsilon_{i}^{k}, \varepsilon_{i}^{k 0}, S_{i}^{k}} \frac{\sum_{k=1}^{3} \sum_{i \neq k} \int_{V}\left\{\sigma_{i k}^{+}\left[\left(\frac{1}{2} S_{i}^{k}+1\right) \varepsilon_{i}^{k}+\frac{1}{2} \varepsilon_{i}^{k 0}\right]-\sigma_{i k}^{-}\left[\frac{1}{2} S_{i}^{k} \varepsilon_{i}^{k}+\frac{1}{2} \varepsilon_{i}^{k 0}\right]\right\} d \mathbf{x}}{\sigma_{Y} \sum_{k=1}^{3} \sum_{i \neq k} \int_{V}\left|\left(S_{i}^{k}+1\right) \varepsilon_{i}^{k}+\varepsilon_{i}^{k 0}\right| d \mathbf{x}}
$$

(inequality because the numerator is increased).

On the other hand substituting a trial field (2.11) with $\left(t \in T_{x}^{k}, i \neq k\right)$

$$
\Lambda_{i}(\mathbf{x}, t)=\left\{\begin{array}{cl}
\frac{1}{2}\left(S_{i}^{k}+2\right) \delta\left(t-t_{x k}^{+}\right)-\frac{1}{2} S_{i}^{k} \delta\left(t-t_{x k}^{-}\right), & \mathbf{x} \in V_{i}^{k} \\
\frac{1}{2} \delta\left(t-t_{x k}^{+}\right)-\frac{1}{2} \delta\left(t-t_{x k}^{-}\right), & \mathbf{x} \in V_{i}^{k 0}
\end{array}\right.
$$

$(\delta(t)$ is the Dirac function) satisfying $(2.12),(2.13)$ (therefore the field is kinematically admissible) into (2.16) we get the same expression as the one after sup in (2.17), so the supremum is reachable and the inequality in (2.17) can be substituted by the equality, that is:

$$
k_{s}^{-1}=\sup _{\varepsilon_{i}^{k}, \varepsilon_{i}^{k 0}, S_{i}^{k}} \frac{\sum_{k=1}^{3} \sum_{i \neq k} \int\left\{\sigma_{i k}^{+}\left[\left(\frac{1}{2} S_{i}^{k}+1\right) \varepsilon_{i}^{k}+\frac{1}{2} \varepsilon_{i}^{k 0}\right]-\sigma_{i k}^{-}\left[\frac{1}{2} S_{i}^{k} \varepsilon_{i}^{k}+\frac{1}{2} \varepsilon_{i}^{k 0}\right]\right\} d \mathbf{x}}{\sigma_{Y} \sum_{k=1}^{3} \sum_{i \neq k} \int_{V}\left|\left(S_{i}^{k}+1\right) \varepsilon_{i}^{k}+\varepsilon_{i}^{k 0}\right| d \mathbf{x}} .
$$

Introduce new functions $\bar{\varepsilon}_{i}^{k}(\mathbf{x})$ such that

$$
\begin{aligned}
& \varepsilon_{i}^{k 0}(\mathbf{x})=S_{i}^{k}(\mathbf{x}) \bar{\varepsilon}_{i}^{k}(\mathbf{x}), \quad S_{i}^{k}(\mathbf{x}) \geq 0, \mathbf{x} \in V_{i}^{k 0} \\
& \left(\bar{\varepsilon}_{i}^{k}(\mathbf{x})=0, \quad \mathbf{x} \in V_{i}^{k}\right) .
\end{aligned}
$$


Functions $S_{i}^{k}(\mathbf{x})$ for $\mathbf{x} \in V_{i}^{k}$ has already been defined in (2.13). Clearly $S_{i}^{k}(\mathbf{x})$ can be arbitrary positive functions. With (2.18), (2.19) can be presented in the form

$$
k_{s}^{-1}=\sup _{\varepsilon_{i}^{k}, \bar{\varepsilon}_{i}^{k}, S_{i}^{k}} \frac{\sum_{k=1}^{3} \sum_{i \neq k} \int_{V}\left[\sigma_{i k}^{+} \varepsilon_{i}^{k}+\frac{1}{2}\left(\sigma_{i k}^{+}-\sigma_{i k}^{-}\right) S_{i}^{k}\left(\varepsilon_{i}^{k}+\bar{\varepsilon}_{i}^{k}\right)\right] d \mathbf{x}}{\sigma_{Y} \sum_{k=1}^{3} \sum_{i \neq k} \int\left(\left|\varepsilon_{i}^{k}\right|+S_{i}^{k}\left|\varepsilon_{i}^{k}+\bar{\varepsilon}_{i}^{k}\right|\right) d \mathbf{x}} .
$$

Denote

$$
\hat{S}_{i}^{k}(\mathbf{x})=S_{i}^{k}(\mathbf{x}) \cdot\left|\varepsilon_{i}^{k}(\mathbf{x})+\bar{\varepsilon}_{i}^{k}(\mathbf{x})\right|, \quad X_{i}^{k}=\int_{V} \hat{S}_{i}^{k}(\mathbf{x}) d \mathbf{x} .
$$

As $S_{i}^{k}$ is arbitrarily positive, $X_{i}^{k}$ is also arbitrarily positive. Substituting (2.21) into $(2.20)$, we get

$$
k_{s}^{-1}=\sup _{\varepsilon_{i}^{k}, \bar{\varepsilon}_{i}^{k}, \hat{S}_{i}^{k}} \frac{\sum_{k=1}^{3} \sum_{i \neq k} \int_{V}\left[\sigma_{i k}^{+} \varepsilon_{i}^{k}+\frac{\left(\sigma_{i k}^{+}-\sigma_{i k}^{-}\right) \hat{S}_{i}^{k}\left(\varepsilon_{i}^{k}+\bar{\varepsilon}_{i}^{k}\right)}{2\left|\varepsilon_{i}^{k}+\bar{\varepsilon}_{i}^{k}\right|}\right] d \mathbf{x}}{\sigma_{Y} \sum_{k=1}^{3} \sum_{i \neq k}\left(\int\left(\left|\varepsilon_{V}^{k}\right| d \mathbf{x}+X_{i}^{k}\right)\right.} .
$$

Denote

$$
U_{i}^{k}=\max _{\mathbf{x} \in V} \frac{\left[\sigma_{i k}^{+}(\mathbf{x})-\sigma_{i k}^{-}(\mathbf{x})\right]\left[\varepsilon_{i}^{k}(\mathbf{x})+\bar{\varepsilon}_{i}^{k}(\mathbf{x})\right]}{2\left|\varepsilon_{i}^{k}(\mathbf{x})+\bar{\varepsilon}_{i}^{k}(\mathbf{x})\right|}
$$

with $\mathbf{x}_{i}^{k}$ being the point where the maximum is reached. From (2.21)-(2.23) we have

$$
k_{s}^{-1} \leq \sup _{\varepsilon_{i}^{k}, X_{i}^{k} ;} \frac{\sum_{k=1}^{3} \sum_{i \neq k}\left(\int_{V} \sum_{k=1}^{3} \sum_{i \neq k}^{+} \varepsilon_{i}^{k} d \mathbf{x}+U_{V}^{k} \cdot X_{i}^{k}\right)}{\left(\left|\varepsilon_{i}^{k}\right| d \mathbf{x}+X_{i}^{k}\right)} .
$$

On the other hand, substituting admissible variables

$$
\begin{aligned}
& \hat{S}_{i}^{k}(\mathbf{x})=X_{i}^{k} \cdot \delta\left(\mathbf{x}-\mathbf{x}_{i}^{k}\right) \\
& \left(\mathbf{x}_{i}^{k}-\text { the maximum point of }(2.23)\right)
\end{aligned}
$$

into (2.22), we get the exact expression after sup in (2.24). Thus, the inequality (2.24) can be substituted by the equality, that is

$$
k_{s}^{-1}=\sup _{\varepsilon_{i}^{k}, X_{i}^{k}} \frac{\sum_{k=1}^{3} \sum_{i \neq k}\left(\int _ { V } \sigma _ { i k } ^ { + } \sum _ { i = 1 } ^ { k } \sum _ { i \neq k } \left(\int_{V}\left(\left|\varepsilon_{i}^{k}\right| d \mathbf{x}+U_{i}^{k} \cdot X_{i}^{k}\right)\right.\right.}{3} .
$$


The expression after sup in (2.26) depends monotonically upon every $X_{i}^{k} \in$ $[0,+\infty)$, so the supremum is reached at $X_{i}^{k}=0$ or $X_{i}^{k}=+\infty$. Hence

$$
k_{s}^{-1}=\max \left\{\sup _{\varepsilon_{i}^{k}} \frac{\sum_{k=1}^{3} \sum_{i \neq k} \int_{V} \sigma_{i k}^{+} \varepsilon_{i}^{k} d \mathbf{x}}{\sigma_{Y} \sum_{k=1}^{3} \sum_{i \neq k} \int_{V}\left|\varepsilon_{i}^{k}\right| d \mathbf{x}}, \max _{k, i} U_{i}^{k}\right\} .
$$

Finally, (2.27) can be given in the form

$$
k_{s}^{-1}=\max \{I, A\},
$$

where

$$
I=\sup _{\sigma^{e} \in \mathcal{L} ; \varepsilon^{p} \in \mathcal{C}} \frac{\sum_{k=1}^{3} \sum_{i \neq k} \int_{V} \max _{t}\left[\sigma_{i}(\mathbf{x}, t)-\sigma_{k}(\mathbf{x}, t)\right] \varepsilon_{i}^{k}(\mathbf{x}) d \mathbf{x}}{\sigma_{Y} \sum_{k=1}^{3} \sum_{i \neq k} \int_{V}\left|\varepsilon_{i}^{k}\right| d \mathbf{x}},
$$

( keep in mind that

$$
\begin{gathered}
\sum_{k=1}^{3} \sum_{i \neq k}\left[\varepsilon_{i}^{k}(\mathbf{x}) \varepsilon^{i}-\varepsilon_{i}^{k}(\mathbf{x}) \varepsilon^{k}\right]=\varepsilon^{p}(\mathbf{x}) \in \mathcal{C} \quad, \\
A=\sup _{\mathbf{x}, t, t, i, k} \frac{\sigma_{i}(\mathbf{x}, t)-\sigma_{k}(\mathbf{x}, t)-\sigma_{i}\left(\mathbf{x}, t^{\prime}\right)+\sigma_{k}\left(\mathbf{x}, t^{\prime}\right)}{2 \sigma_{Y}} .
\end{gathered}
$$

Formulae (2.28)-(2.31) are the required reduced form of (1.3). It contains no time integrals over loading trajectories and clearly reflect the path-independent nature of shakedown theory. Eqs. (2.28)-(2.31) are derived for Tresca material under presumption (2.2). In the general case, as (2.2) is just a class of admissible kinematical fields, (2.18)-(2.31) give an upper bound on $k_{s}$. This bound improves upon that of [10], because the class of admissible fields is broader here.

\section{An illustration}

To illustrate the formulae (2.28)-(2.31) we take a very simple example: a thinwalled circular cylinder subjected to quasistatic axial load $P$ and internal pressure $q$, which vary independently between the limits

$$
\underline{P} \leq P(t) \leq \bar{P}, \quad \underline{q} \leq q(t) \leq \bar{q} .
$$


Correspondingly the axial stress $\sigma_{a}$ and circumferential stress $\sigma_{\phi}$ of the cylinder are (the radial stress $\sigma_{r}$ is negligible)

$$
\begin{array}{ll}
\sigma_{a}(t)=\frac{P(t)}{2 \pi R h}, & \underline{\sigma}_{a}=\frac{\underline{P}}{2 \pi R h}, \quad \bar{\sigma}_{a}=\frac{\bar{P}}{2 \pi R h}, \\
\sigma_{\phi}(t)=\frac{q(t) R}{h}, & \underline{\sigma}_{\phi}=\frac{q}{h}, \quad \bar{\sigma}_{\phi}=\frac{\bar{q} R}{h},
\end{array}
$$

( $R$ is the radius and $h$ - the thickness of the cylinder);

$$
\underline{\sigma}_{a} \leq \sigma_{a}(t) \leq \bar{\sigma}_{a}, \quad \underline{\sigma}_{\phi} \leq \sigma_{\phi}(t) \leq \bar{\sigma}_{\phi}, \quad \sigma_{r}(t) \equiv 0 .
$$

From (2.31),(3.3) one can see that

$$
\begin{aligned}
& \max _{t, t^{\prime}} \frac{\sigma_{a}(t)-\sigma_{\phi}(t)-\sigma_{a}\left(t^{\prime}\right)+\sigma_{\phi}\left(t^{\prime}\right)}{2 \sigma_{Y}}=\frac{\bar{\sigma}_{a}-\underline{\sigma}_{\phi}-\underline{\sigma}_{a}+\bar{\sigma}_{\phi}}{2 \sigma_{Y}}, \\
& \max _{t, t^{\prime}} \frac{\sigma_{a}(t)-\sigma_{r}(t)-\sigma_{a}\left(t^{\prime}\right)+\sigma_{r}\left(t^{\prime}\right)}{2 \sigma_{Y}}=\frac{\bar{\sigma}_{a}-\underline{\sigma}_{a}}{2 \sigma_{Y}}, \ldots,
\end{aligned}
$$

so

$$
A=\max \left\{\frac{\bar{\sigma}_{a}-\underline{\sigma}_{\phi}-\underline{\sigma}_{a}+\bar{\sigma}_{\phi}}{2 \sigma_{Y}}, \frac{\bar{\sigma}_{a}-\underline{\sigma}_{a}}{2 \sigma_{Y}}, \frac{\bar{\sigma}_{\phi}-\underline{\sigma}_{\phi}}{2 \sigma_{Y}}\right\} .
$$

From (2.29),(3.3) follows

$$
\begin{aligned}
\max _{t}\left(\sigma_{a}-\sigma_{\phi}\right) \varepsilon & =\max \left\{\left(\bar{\sigma}_{a}-\underline{\sigma}_{\phi}\right) \varepsilon,\left(\underline{\sigma}_{a}-\bar{\sigma}_{\phi}\right) \varepsilon\right\} \\
& =|\varepsilon| \cdot \max \left\{\left|\bar{\sigma}_{a}-\underline{\sigma}_{\phi}\right|,\left|\underline{\sigma}_{a}-\bar{\sigma}_{\phi}\right|\right\}=|\varepsilon| \cdot \hat{\sigma}_{a \phi}, \\
\max _{t}\left(\sigma_{a}-\sigma_{r}\right) \varepsilon_{a}^{r} & =\max \left\{\bar{\sigma}_{a} \varepsilon_{a}^{r}, \underline{\sigma}_{a} \varepsilon_{a}^{r}\right\} \\
& =\left|\varepsilon_{a}^{r}\right| \cdot \max \left\{\left|\bar{\sigma}_{a}\right|,\left|\underline{\sigma}_{a}\right|\right\}=\left|\varepsilon_{a}^{r}\right| \cdot \hat{\sigma}_{a}, \\
\max _{t}\left(\sigma_{\phi}-\sigma_{r}\right) \varepsilon_{\phi}^{r} & =\left|\varepsilon_{\phi}^{r}\right| \cdot \max \left\{\left|\bar{\sigma}_{\phi}\right|,\left|\underline{\sigma}_{\phi}\right|\right\}=\left|\varepsilon_{\phi}^{r}\right| \cdot \hat{\sigma}_{\phi}, \ldots .
\end{aligned}
$$

Thus

$$
I=\sup _{\varepsilon^{p} \in C} \frac{\hat{\sigma}_{a \phi}\left(\left|\varepsilon_{a}^{\phi}\right|+\left|\varepsilon_{\phi}^{a}\right|\right)+\hat{\sigma}_{a}\left(\left|\varepsilon_{a}^{r}\right|+\left|\varepsilon_{r}^{a}\right|\right)+\hat{\sigma}_{\phi}\left(\left|\varepsilon_{\phi}^{r}\right|+\left|\varepsilon_{r}^{\phi}\right|\right)}{\sigma_{Y}\left(\left|\varepsilon_{a}^{\phi}\right|+\left|\varepsilon_{\phi}^{a}\right|+\left|\varepsilon_{a}^{r}\right|+\left|\varepsilon_{r}^{a}\right|+\left|\varepsilon_{\phi}^{r}\right|+\left|\varepsilon_{r}^{\phi}\right|\right)} .
$$

In this simple example the condition $\varepsilon^{p} \in \mathcal{C}$ imposes no restriction on $\varepsilon_{a}^{\phi}, \varepsilon_{\phi}^{a}$, $\varepsilon_{a}^{r}, \varepsilon_{r}^{a}, \varepsilon_{\phi}^{r}, \varepsilon_{r}^{\phi}$, therefore it is obvious that (consult also the deduction of (2.27) from $(2.26))$

$$
\begin{aligned}
I & =\frac{1}{\sigma_{Y}} \cdot \max \left\{\hat{\sigma}_{a \phi}, \hat{\sigma}_{a}, \hat{\sigma}_{\phi}\right\} \\
& =\frac{1}{\sigma_{Y}} \cdot \max \left\{\bar{\sigma}_{a}-\underline{\sigma}_{\phi}, \bar{\sigma}_{\phi}-\underline{\sigma}_{a}, \bar{\sigma}_{a},-\underline{\sigma}_{a}, \bar{\sigma}_{\phi},-\underline{\sigma}_{\phi}\right\} .
\end{aligned}
$$


Finally

$k_{s}^{-1}=\max \{I, A\}=\frac{1}{\sigma_{Y}} \cdot \max \left\{\bar{\sigma}_{a}-\underline{\sigma}_{\phi}, \bar{\sigma}_{\phi}-\underline{\sigma}_{a}, \bar{\sigma}_{a},-\underline{\sigma}_{a}, \bar{\sigma}_{\phi},-\underline{\sigma}_{\phi}\right\}$.

This simple case serves only to illustrate the formulae (2.28)-(2.31). Application of the formulae to solve more complex problems should be the subject of further studies.

The kinematic approach is expected to have some advantage over the static one in the development of numerical methods for applications. The strain-space formulation is naturally associated with the most usual displacement-based spacial discretization scheme of the finite-element method and the strain control seems more appropriate for perfectly plastic solids. So it is worth to give further attention to the approach.

This work is supported by the Program of Basic Research in Natural Science.

\section{REFERENCES}

1. Drucker D. C., Prager W., Greenberg H. J. Extended limit design theorems for continuous media. Q. Appl. Math. 9 (1951), 381-389.

2. Hill $\mathrm{R}$. On the state of stress in a plastic-rigid body at the yield point. Phil. Mag. 42 (1951), 868-875.

3. Koiter W. T. General theorems for elastic-plastic solids. In Progress in solids mechanics (Ed. I.N. Sneddon and R. Hill), North-Holland, Amsterdam (1963), $165-221$.

4. Corradi L., Maier G. Dynamic inadaptation theorem for elastic-perfectly plastic continua. J. Mech. Phys. Solids 22 (1974), 401-413.

5. Ceradini G. Dynamic shakedown in elastic-plastic bodies. J. Engng. Mech. Div. Proc. ASCE 106 (1980), 481-499.

6. Pham D. C. Dynamic shakedown and a reduced kinematic theorem. Int. J. Plasticity 12 (1996), 1055-1068.

7. Gokhfeld D. A. Some problems of shakedown of plates and shells. Proc. VI-th Soviet Conf. Plates Shells, Nauka (1966), 284-291.

8. Sacwzuk A. Evaluation of upper bounds to shakedown loads of shells. J. Mech. Phys. Solids 17 (1969), 291-301.

9. König J. A., Shakedown of elastic-plastic structures. Elsevier Amsterdam (1987)

10. Pham D. C., Stumpf H. Kinematical approach to the shakedown analysis of some structures. Q. Appl. Math. 52 (1994), 707-719. 
11. Pham D. C. Reduced forms of shakedown kinematic theorem for elastic-perfectly plastic bodies. Proc. R. Soc. Lond. A 453 (1997), 2259-2269.

12. Pham D. C. An upper bound kinematic approach to the shakedown analysis of structures. Mecanica 34 (1999), 49-56.

Received November 2, 2000

\section{MỘT DANGG GIẢN YẾU CỦA ĐỊNH LÝ THíCH NGHI ĐỘNG}

Một dạng giản yếu của định lý thích nghi động được xây dựng cho vật liệu Tresca. Biểu thức nhận được tương đương với định lý khởi thủy khi các hướng chính của biến dạng dẻo không thay đổi trong các chu trình đặt tải. 